\title{
Optimal Linear Coding Schemes for the Secure Decentralized Pliable Index Coding Problem
}

\author{
Tang Liu and Daniela Tuninetti, \\ University of Illinois at Chicago, Chicago, IL 60607 USA, \\ Email: tliu44, danielateuic.edu
}

\begin{abstract}
We study the secure decentralized Pliable Index CODing (PICOD) problem with circular side information sets at the users. The security constraint forbids every user to decode more than one message while decentralized setting means there is no central transmitter in the system. Compared to the secure but centralized version of the problem, a converse bound from one of our previous works showed a factor of three difference in optimal code length under the constraint of linear encoding. In this paper, we first list the linearly infeasible cases, that is, problems where no linear code can simultaneously achieve both correctness/decodability and security. Then, we propose linear coding schemes for all remaining cases and show that their attained code length is to within an additive constant gap from our converse bound.
\end{abstract}

\section{INTRODUCTION}

In this paper we study the Secure Decentralized Pliable Index CODing (SD-PICOD) problem. PICOD is a variant of the Index Coding (IC) problem, motivated by broadcast systems where users have message side information sets to help reduce the number of transmissions needed to satisfy the users' demands [2].

Index Coding: The traditional IC setting consists of $m$ messages, one central transmitter, and $n$ users. The transmitter and the users are connected by an error-free broadcast channel. Each user has some messages locally stored as its side information set and has one pre-determined message to decode. The structure of the side information sets and the desired messages are known to the transmitter and all the users. The transmitter broadcasts coded symbols to all users. The users decode based on the received coded symbols and their own side information set. The goal for IC is to find the smallest code-length / number of transmitted coded symbols such that all users can decode correctly their desired messages.

Pliable Index Coding: PICOD is a variant of IC motivated by the scenarios where the desired message at the users is not be pre-determined [3] such as for example streaming services and online advertisement systems. In PICOD a user is satisfied whenever it can correctly decode at least one message that is not in its side information set. Therefore, the transmitter can leverage the freedom of choosing the desired messages for the users so as to reduce the code-length. Compared to the IC with the same number of users and the same side information sets, PICOD needs less number of transmissions to satisfy all the users [9].

Decentralized Problems: The decentralized IC problem is motivated by peer-to-peer communication systems where there is no central transmitter and instead coded symbols are generated by the users based on their side information and sent through a common time-sharing noiseless broadcast channel. The goal again is to find the minimal code-length that allows every user to correctly decode its desired message. Under linear encoding constraint, the minimum code-length of the decentralized IC is shown to be no more than twice that of its centralized counterpart [1]. The pliable version of decentralized IC was studied in [4], where information theoretical bounds on the optimal code-length were given for several cases. For the solved cases, the multiplicative gap between centralized and decentralized PICOD is usually much less than two.

Secure Centralized Problems: Security in IC means that the users can only decode their desired message while all other messages that are not in their side information set must remain unknown to the users. The secure IC problem was first proposed in [7], where private one-time-pad keys were used to meet the security demand. A weaker definition of security which can be achieved without security keys has also been discussed in [7] and has been extended to PICOD setting [8]. Achievable and converse bounds for the case of circular side information structure and linear encoding were derived in [5].

Secure Decentralized Problems: In this paper we are interested in the secure and decentralized setting. Recently, the secure PICOD problem in [5] has been extended to the decentralized setting in [6], where a converse bound under the constraint of linear encoding showed a multiplicative gap of roughly three between the secure centralized and decentralized versions of PICOD. This gap is strictly larger than the one between the centralized and decentralized versions of IC without security which equals two [1].

Contributions: We continue here the line of study initiated in [6], where we found: (i) there are infeasible cases when $m$, the number of users, is odd, and (ii) the proposed achievable scheme does not match the converse bounds (in a multiplicative or additive gap sense) in general. This paper completely answers the question posed in [6] by (i) providing a complete list of infeasible cases, which shows that most cases when $m$ is odd are actually feasible, and (ii) showing achievable schemes for all feasible cases. We conclude that the new schemes achieve the converse bounds in [6] to within an additive constant gap.

Paper Organization: The rest paper is organized as follows. Section II introduces the problem. Section III summa- 
rizes the main results in this paper. In Section IV] we prove the infeasible cases. In Section $\nabla$ we illustrate the main ideas for the achievable scheme by way of examples to provide the intuitions for the general scheme. Section VI concludes the paper. The details of the proofs can be found in the Appendix.

\section{PROBLEM FORMULATION}

We consider the $(m, s)$ secure decentralized PICOD problem with circular side information structure at the users. The system consists of $m$ messages and $m$ users. The messages are vectors of length $\kappa \in \mathbb{N}$ of independent and uniformly distributed bits. $\mathcal{W}:=\left\{w_{1}, \ldots, w_{m}\right\}$ denotes the set of all messages. $W_{A}:=\left\{w_{i}, i \in A\right\}$ denotes the set of messages with indices in set $A . \mathcal{U}:=\left\{u_{1}, \ldots, u_{m}\right\}$ denotes the set of the users. User $u_{i}$ has message $W_{A_{i}}$ as its side information, where $A_{i}=\{i, i-1 \ldots, i-s+1\}$. The entries in the side information set are intended modulo $m$. The collection $\mathcal{A}=\left\{A_{1}, \ldots, A_{m}\right\}$ is globally known to all users. The coded symbols are generated by the users based on their side information set. The encoding function at user $i$ is

$$
x^{\kappa \ell_{i}}:=\mathbf{E N C}_{i}\left(W_{A_{i}}, \mathcal{A}\right), i \in[m],
$$

where $\ell_{i} \in \mathbb{N}$ is the code length. The overall transmission is represented by the vector $x^{\kappa \ell}=\left\{x^{\kappa \ell_{1}}, \ldots, x^{\kappa \ell_{1}}\right\}$ with total normalized length $\ell=\sum_{i \in[m]} \ell_{i}$.

Each user must correctly acquire a message that is not in its side information set. The decoding function at user $i$ is

$$
\widehat{w}_{i}:=\mathbf{D E C}_{i}\left(x^{\kappa \ell}, W_{A_{i}}\right), i \in[m] .
$$

The decoding is correct if $\widehat{w}_{i}=w_{d_{i}}$ for some $d_{i} \in[m] \backslash A_{i}$.

The security constraint requires user $i$ decodes no more than one message. Specifically, we have $I\left(w_{j} ; x^{\kappa \ell}, W_{A_{i}}\right)=0$ for all $j \in[m] \backslash\left\{A_{i} \cup\left\{d_{i}\right\}\right\}$ and $i \in[m]$.

The goal is to find the smallest $\ell$ so that all users can correctly decode and the security constraints are satisfied.

\section{MAin CONTRIBUTIONS}

For the $(m, s)$ secure decentralized PICOD with circular side information at the users, the converse bound in [6] gives

$$
\ell^{*} \geq \begin{cases}\frac{m}{s}, & \frac{m}{m-s} \in \mathbb{Z}, \\ \frac{3 m}{2 s}, & \frac{m}{m-s} \notin \mathbb{Z}, \text { linear encoding, } m>2 s, \\ 2, & \frac{m}{m-s} \notin \mathbb{Z}, \text { linear encoding, } m<2 s .\end{cases}
$$

In [6] we found several cases where the problem is infeasible, that is, no linear scheme exists such that every user can decode one and only one message outside its side information set. For such cases, the converse bound in (3) is not tight. In this paper, we first give a list of infeasible cases and show scheme that attain the converse bound in (3) to within an additive constant gap. Specifically, we have the following theorems.

Theorem 1 (All infeasible cases). The secure $(m, s)$ decentralized PICOD with circular side information sets and linear encoding is infeasible if

1) $m \geq 2 s+1, s=1$ or 2 ;
2) Odd $m \geq 7, s=3$ or 4 ;

3) Odd $m, s=m-2$.

Theorem 2 (Achievability to within an additive gap of 7). For the $(m, s)$ secure decentralized PICOD with circular shift side information sets that are not described in Theorem 1$]$ the following is attainable

$$
\ell^{*} \leq \begin{cases}\frac{m}{s}, & \frac{m}{m-s} \in \mathbb{Z}, \\ \frac{3 m}{2 s}+3, & \frac{m}{m-s} \notin \mathbb{Z}, \text { linear encoding, } m>2 s, \\ 2+5, & \frac{m}{m-s} \notin \mathbb{Z}, \text { linear encoding, } m<2 s .\end{cases}
$$

\section{Proof of Theorem 1}

The two infeasible cases in the centralized secure PICOD setting in [5], namely (i) Odd $m, s=1$, and (ii) Odd $m$, $s=m-2$, are infeasible also in the decentralized setting. In [6] we showed the infeasiblility in the decentralized setting of (iii) Even $m \geq 3, s=1$, (iv) $m \geq 5, s=2$, (v) Odd $m$, $s=3$. In the rest of this section we prove the last infeasible case (vi) odd $m \geq 7, s=4$, and we split it into two cases.

\section{A. Case Odd $m \geq 9, s=4$}

Assume we have an achievable scheme that satisfies all constraints. Since here $m>2 s$, from [5, Proposition 1], one transmission involves at least 2 messages. The number of messages in one transmission is either 2 or 3 , since involving $s=4$ consecutive messages in one transmission is insecure as shown in [6]. For a linear code, the user can decode its desired message if there exists one linear combination of the messages such that all the messages but its desired message that are involved in the linear combination are in the user's side information set. Therefore, if two transmissions do not satisfy one user and involve no messages in common, the linear combination of these two transmissions will not satisfy the user. Each transmission involving 2 or 3 consecutive messages satisfies 2 users. Each transmission involving 2 nonconsecutive messages satisfies 4 users. Thus if no transmissions have common messages the total number of satisfied users are even, which contradicts to the condition that $m$ is odd. Therefore, there must exists two transmissions that have messages in common. We consider the following three sub-cases.

a) Both transmissions involve 2 messages: There exists a user that can decode the common message by one transmission then decode another message by the other transmission. Therefore the user can decode two messages and violates the security constrain.

b) One transmission involves 2 messages and the other 3 messages: Let $g($.$) denote a linear combination of its$ argument. We have the following cases:

1) $g_{1}\left(W_{4,5}\right)$ and $g_{2}\left(W_{4,5,6}\right)$ : the user with side information $W_{1,2,3,4}$ can decode $W_{5,6}$, which is insecure;

2) $g_{1}\left(W_{4,5}\right)$ and $g_{2}\left(W_{5,6,7}\right)$ : the user with side information $W_{6,7,8,9}$ can decode $W_{4,5}$, which is insecure;

3) $g_{1}\left(W_{4,6}\right)$ and $g_{2}\left(W_{4,5,6}\right)$ : the user with side information $W_{1,2,3,4}$ can decode $W_{5,6}$, which is insecure;

4) $g_{1}\left(W_{4,6}\right)$ and $g_{2}\left(W_{5,6,7}\right)$ : the user with side information $W_{6,7,8,9}$ can decode $W_{4,5}$, which is insecure; 
5) $g_{1}\left(W_{3,5}\right)$ and $g_{2}\left(W_{5,6,7}\right)$ : the user with side information $W_{6,7,8,9}$ can decode $W_{3,5}$, which is insecure.

c) Both transmissions involve 3 consecutive messages:

We have the following cases:

1) $g_{1}\left(W_{4,5,6}\right)$ and $g_{2}\left(W_{4,5,6}\right)$ : a linear combination of these two transmissions can generate a linear combination of $W_{4,5}$. This case is insecure as shown in case 1 of IV-A0b;

2) $g_{1}\left(W_{4,5,6}\right)$ and $g_{2}\left(W_{5,6,7}\right):$ the user with side information $W_{2,3,4,5}$ can decode $W_{6,7}$, which is insecure;

3) $g_{1}\left(W_{4,5,6}\right)$ and $g_{2}\left(W_{6,7,8}\right):$ a linear combination of these two transmissions can generate a linear combination of $W_{4,5,6,7,8}$. This is the only case that does not violate the security constraint. However, this case does not allow any new users to decode. Therefore, the number of satisfied users is the the sum of the number of users satisfied by each transmission, which even. This contradicts the condition that $m$ is odd.

This concludes the proof that the case odd $m \geq 9$ and $s=4$ is infeasible.

\section{B. Case $m=7, s=4$}

Assume we have an achievable scheme that satisfies all the constraints. If all the transmissions are linear combinations of at least 2 messages, the argument for the case $s=4$, odd $m \geq 9$ holds and thus the case is infeasible. Therefore, it is enough to show that the case $s=4, m=7$ is insecure if there is one transmission that involves only one message. Without loss of generality, assume one transmission is a linear function of $w_{4}$. Users $u_{1}, u_{2}, u_{3}$ will decode $w_{4}$ since they do not have it in their side information set. User $u_{3}$ have all the side information of user $u_{4}$ after decoding. Therefore, the desired message of user $u_{4}$ needs to be inside the side information of $u_{3}$. The desired message of $u_{4}$ can only be $w_{7}$. The the argument applies to $u_{7}$ and its desired message can only be $w_{1}$ Thus, only the desired message of user $u_{5}$ and user $u_{6}$ are not fixed yet. Consider the following cases for the desired message of user $u_{5}$.

a) $d_{5}=1$ : User $u_{5}$ can mimic $u_{4}$ and decode $w_{7}$.

b) $d_{5}=7$ : There must exists a linear combination of codewords that is a linear combination of messages $W_{2,3,5,7}$ by the decoding condition of the linear index code, where $w_{7}$ has non-zero coefficient. Let $k \in\{2,3,5\}$ be the largest index in $\{2,3,5\}$ so that $w_{k}$ has non-zero coefficient in said linear combination. User $u_{k-1}$ can decode $w_{k}$ when $k=2,3$, user $u_{3}$ can decode $w_{5}$ when $k=5$. Since these users already have other desired messages, they now decode more than one messages.

c) $d_{5}=6$ : There must exist a linear combination of codewords that is a linear combination of messages $W_{2,3,5,6}$ by the decoding condition of the linear index code, where the coefficient of $w_{6}$ is non-zero. Therefore, there exists one transmission involving $w_{6}$. We consider all possible linear combinations that involve $w_{6}$.

- Linear combination of $w_{6}$, or $W_{4,6}$, or $W_{1,6}$, or $W_{6,7}$, or $W_{1,6,7}$. It will allow user $u_{3}$ to decode parts of $w_{6}$.
- Linear combination of $W_{5,6}$ or $W_{4,5,6}$. It will allow user $u_{2}$ to decode of $w_{5}$ since user $u_{2}$ has $w_{4}$ as the desired message.

Therefore, the case $s=4, m=7$ is infeasible.

Overall, we conclude that the case $s=4$, odd $m \geq 7$ is infeasible. This completes the proof of the infeasible case list in Theorem 1

\section{Proof of Theorem 2}

In this section, we give examples to demonstrate the key ideas in our achievable schemes. The details on the general case can be found in Appendices.

\section{A. Case $\frac{m}{m-s} \in \mathbb{Z}: \ell^{*}=\frac{m}{s}$}

The information theoretical converse was derived in [6] for the centralized case. The achievable scheme is the one for the decentralized PICOD without security constraint discussed in [4], which satisfies the security constraint because for each user, among all the messages that are involved in the encoding function, there is one and only one message that is not in its side information set. Therefore, the scheme is also information theoretically optimal with an additional security constraint.

\section{B. Case $\frac{m}{m-s} \notin \mathbb{Z}, m>2 s: \ell \leq \frac{3 m}{2 s}+3$}

The converse bound in this regime is $\frac{3 m}{2 s}[5]$. Therefore, in order to have an achievable scheme that is optimal to within an additive constant gap, we aim to satisfy on average $\frac{2 s}{3}$ users in one transmission.

It has been shown that, for the cases where $\frac{m}{2 s} \in \mathbb{Z}$, an optimal secure centralized scheme is $\left\{w_{1+2 s k}+w_{2+2 s k}, w_{3+2 s k}+\right.$ $\left.w_{s-2+2 s k}, w_{s-3+2 s k}+w_{s-4+2 s k}\right\}, k \in\left\{0,1, \ldots, \frac{m}{2 s}-1\right\}$, for a total $\frac{3 m}{2 s}$ transmissions [6]. In this section, by ways of examples, we show how this scheme can be extended to all feasible cases in the regime $\frac{m}{m-s} \notin \mathbb{Z}, m>2 s$.

To make the exposition easier, we shall use the case $m=26$ in the following and represent the scheme in a "matrix" form figures. In the figure, a row with $\mathbf{X}$ 's represents a transmission that is a linear combination of the messages marked by the $\mathbf{X}$. A row with U's shows the users that are satisfied by the transmission shown by the row right above. The user represented by the $\mathbf{U}$ in position $i$ is the user with side information set $W_{A_{i}}=\left\{w_{i-s+1}, \ldots, w_{i}\right\}$.

a) Case $m=26, s=6$ : The scheme is illustrated in Fig. 11 We have two groups of $2 s=12$ users each. Six transmissions are used to satisfy the 24 users in these groups. The transmissions are $w_{1}+w_{2}, w_{3}+w_{7}, w_{8}+w_{9}, w_{13}+$ $w_{14}, w_{15}+w_{19}, w_{20}+w_{21}$. The two remaining users are satisfied by the last transmission $w_{22}+w_{23}+w_{24}+w_{25}+w_{26}$. The total number of transmissions is $\ell=7$.

b) Case $m=26, s=10$ : We have one group of $2 s=20$ users, and 6 remaining users. The transmissions and the users that are satisfied by each transmission are illustrated in Fig. 2 Three transmissions satisfy the 20 users' group. The transmissions are $w_{1}+w_{2}, w_{3}+w_{12}, w_{13}+w_{14}$. For the remaining users, we use two transmissions $w_{15}+w_{17}+w_{18}+w_{19}+w_{20}+$ $w_{21}+w_{22}$ and $w_{18}+w_{19}+w_{20}+w_{21}+w_{22}+w_{23}+w_{25}$, 


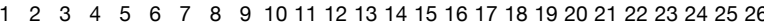

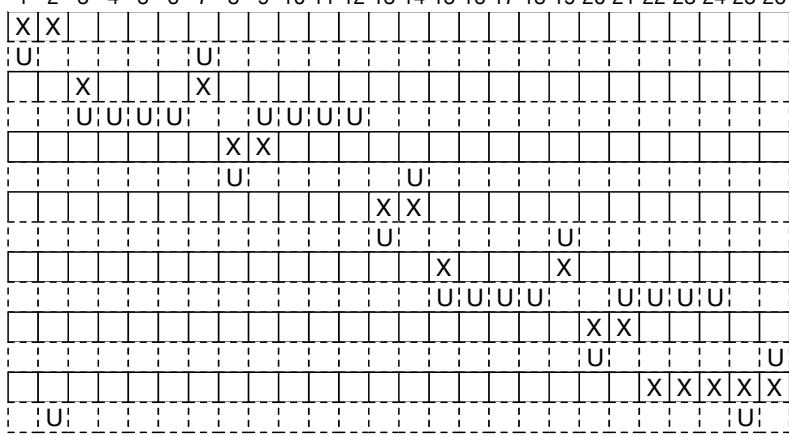

Fig. 1: Achievable scheme for $m=26, s=6$.

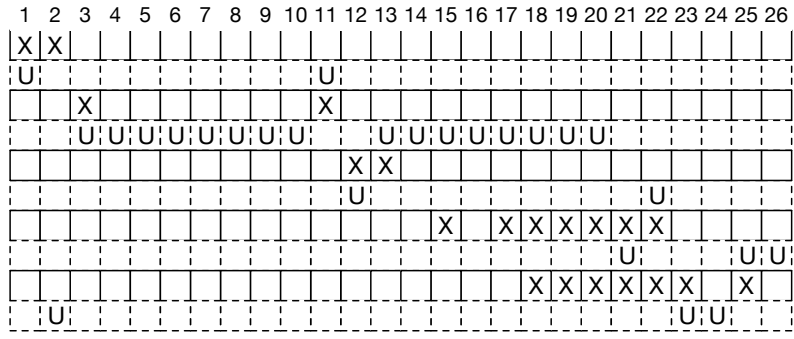

Fig. 2: Achievable scheme for $m=26, s=10$.

Each satisfying three of the remaining users. The total number of transmissions is $\ell=5$.

Remark 1. The key ideas here are as follows. In the regime $m>2 s$ we use the scheme that satisfies $2 s$ users by using three transmissions. In the first step, we group the users into disjoint groups of size $2 s$ and satisfy the users in each group with 3 transmissions. In the second step, we satisfy the remaining users, which are less than $2 s$. Our scheme guarantees that the number of transmissions needed to satisfy the remaining users is a constant that does not grow with the system parameter $(m, s)$. Therefore, for the feasible cases, the proposed scheme can achieve the converse bound to within an additive constant gap that equals the number of transmissions in the second step.

C. Case $\frac{m}{m-s} \notin \mathbb{Z}, s<m<2 s$ : $\ell \leq 9$

In this regime the scheme in [6] does not work because the number of users is $m<2 s$, thus no group of size $2 s$ users can be formed. Here we aim for satisfying all users with a constant number of transmissions that does not grow with the system parameters $(m, s)$. We treat two sub-cases separately.

1) Subcase $\frac{m}{m-s} \notin \mathbb{Z}, s<m \leq \frac{3 s}{2}$ : In this case we consider the complement of the side information set of every user, which is of size $m-s$. The proposed scheme guarantees that among every consecutive $m-s$ messages, the codewords contain one and only one message that is linearly independent of the remaining $m-s-1$ messages. In the following, we provide examples to demonstrate the scheme. The detailed proof can be found in Appendix B

a) Case $m=26, s=20$ : The codewords contain two parts:
$122 \quad 3 \quad 4 \quad 5 \quad 6 \quad 7 \quad 8 \quad 9 \quad 1011121314151617181920212223242526$

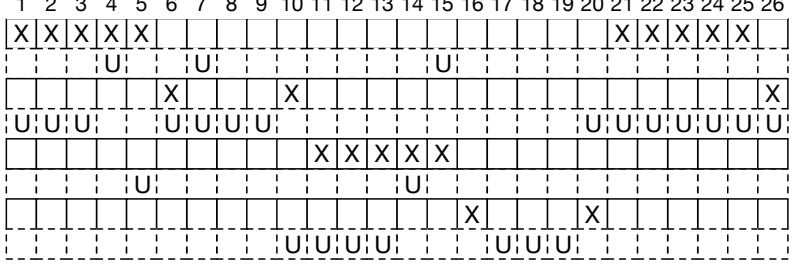

Fig. 3: Achievable scheme for $m=26, s=20$.

\begin{tabular}{|c|c|c|c|c|c|c|c|c|c|c|c|c|c|c|c|c|c|c|}
\hline & & & $\mathrm{X}$ & & & & & & & & & & & & & & & \\
\hline U:U & נIU & 1 & & & & & & & & & & & & & & & & UIU \\
\hline \begin{tabular}{l|l}
$\mathrm{x}$ \\
$\mathrm{x}$
\end{tabular} & $x$ & & & $\mathrm{X}$ & & & $X$ & & & & & & & & & $\mathrm{X}$ & & \\
\hline & & & & $\bar{U}$ & $\mathrm{JIU:}$ & IU: & & & & & נ:U & $\mathbf{U}$ & U: & U U U & & & & \\
\hline & & & & & & & & $x$ & & $x \mid x$ & & & & & & & & \\
\hline & & & $U$ & & iUi & & & & & $U_{i}^{\prime}$ & & & & & & & & \\
\hline & & & & & & & & & & & & & $\mathrm{X}$ & & $\mathrm{X}$ & & $\mathrm{X}$ & \\
\hline & & & & & & & $\underline{U}$ & 10 & & & & & 4 & 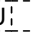 & & U & 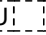 & \\
\hline
\end{tabular}

Fig. 4: Achievable scheme for $m=26, s=19$.

- Part A with the structure $w_{i+1}+\ldots+w_{i+(m-s-1)}$, $w_{m-s}+w_{i+2(m-s-1)}$. It takes totally $2 k_{1}(m-s-1)$ consecutive messages, where $k_{1} \geq 2, k_{1} \in \mathbb{Z}$.

- Part B with the structure $w_{j}, w_{j+1}+\ldots+w_{j+(m-s)}$. It takes totally $k_{2}(m-s)$ consecutive messages, where $k_{2} \geq 0, k_{2} \in \mathbb{Z}$.

Therefore, this scheme works for the case $m=k_{1} 2(m-s-$ $1)+k_{2}(m-s)$, where $k_{1} \geq 2, k_{2} \geq 0, k_{1}, k_{2} \in \mathbb{Z}$.

In the case of $s=20, m=26=2(10)+6$, the proposed scheme takes 4 transmissions: 1) $w_{1}+w_{2}+w_{3}+w_{4}+w_{5}+$ $\left.\left.w_{21}+w_{22}+w_{23}+w_{24}+w_{25} ; 2\right) w_{6}+w_{1} 0+w_{26} ; 3\right) w_{11}+$ $w_{12}+w_{13}+w_{14}+w_{15}$; 4) $w_{16}+w_{20}$, as shown in Fig. 3 .

For the cases where $m \neq k_{1} 2(m-s-1)+k_{2}(m-s), \forall k_{1} \geq$ $2, k_{2} \geq 0, k_{1}, k_{2} \in \mathbb{Z}$, we propose the following examples to show how the scheme can be modified.

b) Case $m=26, s=19$ : In this case the proposed scheme takes 4 transmissions: 1) $w_{5}$;2) $w_{2}+w_{3}+w_{4}+w_{6}+$ $w_{11}+w_{22}$; 3) $w_{12}+w_{13}+w_{14}+w_{15}$; 4) $w_{18}+w_{21}+w_{24}$; as shown in Fig. 4. One can check that for every 7 consecutive messages, there exists one and only one message that is linearly independent of the other 6 messages. The scheme can be seen as a modified version of the scheme in Section $\mathrm{V}-\mathrm{C} 1 \mathrm{a}$ the part from $w_{6}$ to $w_{17}$ is a modified Part A; the part from $w_{25}$ to $w_{5}$ is a modified Part $\mathbf{B}$; and the rest is a new structure that combines these two modified pieces.

c) Case $m=26, s=18:$ In this case the proposed scheme takes 4 transmissions: 1) $w_{1}+w_{4}+w_{5}+w_{6}+w_{7}$; 2) $w_{8}+w_{13}$; 3) $w_{15}+w_{16}+w_{17}+w_{18}+w_{19}$; 4) $w_{22}+w_{26}$, as shown in Fig. 5 One can check for every 8 consecutive messages, there exists one and only one message that is linear independent from the other 7 messages. The scheme can be seen as a modified version of the scheme used in Section V-C1a: the part from $w_{1}$ to $w_{14}$ is a modified part $\mathrm{A}$; the part from $w_{15}$ to $w_{26}$ is a modified and shrunk part $\mathrm{A}$.

Remark 2. The key ideas here are as follows. In this regime our proposed scheme is designed such that every consecutive 


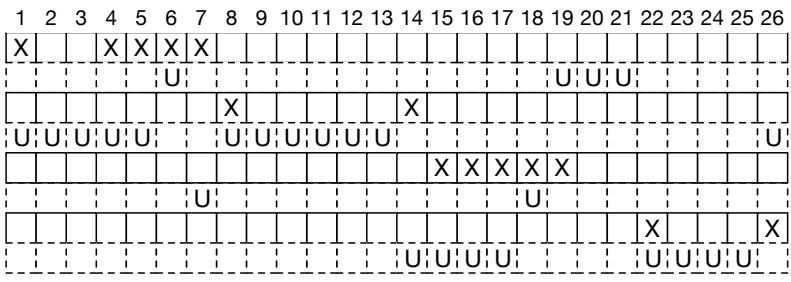

Fig. 5: Achievable scheme for $m=26, s=18$.

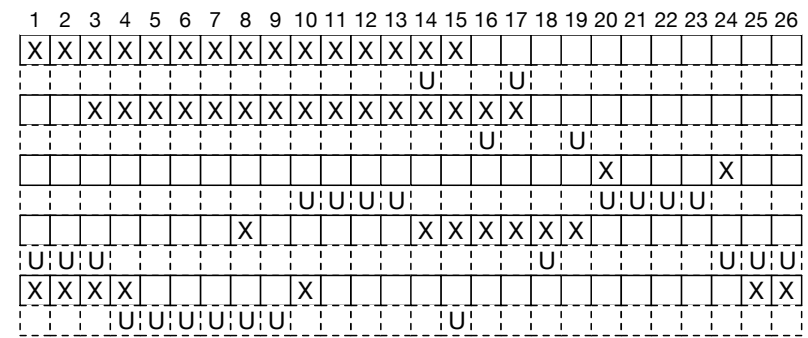

Fig. 6: Achievable scheme for $m=26, s=16$.

$m-s$ messages have one and only one messages that is linearly independent of the rest. The scheme treats the users is a "sliding window" fashion, where the window is of size $m-s$. The scheme for case $m>2 s$ can not be extended straightforwardly in this case because to satisfy users less than $2 s$ the scheme has transmissions that takes more than $2 s$ consecutive messages. This is not an issue in the case $m>2 s$. But when $m<2 s$ this means the transmissions will have messages in common as the indices of the messages are in modulo $m$. The common messages among the transmissions will cause insecurity as it could allow one user to decode more than one message. The security constraint does not hold anymore. The sliding window idea does not have such issues when considering about the security constraint.

2) Subcase $\frac{m}{m-s} \notin \mathbb{Z}, \frac{3 s}{2}<m<2 s$ : Here we propose the scheme that can be thought of as a combination of the ideas we presented in Sections $\mathrm{V}-\mathrm{B}$ and $\mathrm{V}-\mathrm{C} 1$. That is, we first use a finite number of transmissions to satisfy a finite number of users with the scheme in [6]. Then, we use a scheme similar to the one proposed for the case $m>2 s$ in Sections $\mathrm{V}$-B for the remaining users. The total number of transmissions is thus finite and the scheme achieves the converse bound to within a constant gap. The following example illustrates the proposed scheme. The detailed proof of the scheme can be found in Appendix C

a) Case $m=26, s=16$ : The first two transmissions are: 1) $\left.w_{1}+\cdots+w_{15} ; 2\right) w_{3}+w_{17}$. Four users are satisfied by these two transmissions. The remaining users are grouped into 6 groups and are satisfied by 3 transmissions, where each transmission aims to satisfy 2 groups. The transmissions are: 3) $w_{20}+w_{24}$;4) $w_{8}+w_{14}+\cdots+w_{19}$; 5) $w_{1}+\cdots+4+w_{10}+$ $w_{25}+w_{26}$. The transmissions and the corresponding satisfied users are shown in Fig. 6. The scheme uses 5 transmissions to satisfy all users.

We thus proposed schemes that are to within a constant gap from the converse bound in (3). We summarize all cases and the corresponding bounds in Table [

TABLE I: All cases and the corresponding achievable schemes. $m_{\mid a}$ represents $m \bmod a$, where $a$ is an integer; "infea." is the abbreviation of "infeasible".

\begin{tabular}{|c|c|c|c|}
\hline Condition & Subcase & Converse & $\ell$ \\
\hline$\frac{m}{m-s} \in \mathbb{Z}$ & all & $m / s$ & $m / s$ \\
\hline & $m_{\mid 2 s}=0$ & & $\frac{3 m}{2 s}$ \\
\hline$\underline{m} \notin \mathbb{Z}$, & $m_{\mid 2 s}=1$ & & $\frac{3 m}{2}+\frac{4 s-3}{2}$ \\
\hline $\begin{array}{l}m-s \\
m-4 \geq\end{array}$ & $m_{12 s}=2$ & $\frac{3 m}{0}$ & $\frac{2 s}{3 m}+s-3$ \\
\hline$s \geq 5$ & $m \mid 2 s-2$ & & $\begin{array}{c}\overline{2 s}+\bar{s} \\
3 m+6 s-3\end{array}$ \\
\hline$m>2 s$ & $m_{\mid 2 s}=3$ & & $\frac{2 s}{2 s}+\frac{2 s}{2 s}$ \\
\hline & $m_{\mid 2 s} \in[4: s]$ & & $\frac{3 m}{2 s}+\frac{4 s-3 m_{\mid 2 s}}{2 s}$ \\
\hline & $m_{\mid 2 s} \in[s+1: 2 s-2]$ & & $\frac{3 m}{2 s}+\frac{6 s-3 m_{\mid 2 s}}{2 s}$ \\
\hline & $m_{\mid 2 s}=2 s-1$ & & $\frac{3 m}{2 s}+\frac{2 s+3}{2 s}$ \\
\hline $\begin{array}{l}\frac{m}{m-s} \notin \mathbb{Z}, \\
m-4 \geq\end{array}$ & $s<m \leq 3 s / 2$ & 2 & $\leq 4$ \\
\hline $\begin{array}{l}s \geq 5 \\
m<2 s\end{array}$ & $3 s / 2<m<2 s$ & & $\leq 9$ \\
\hline \multirow{6}{*}{ 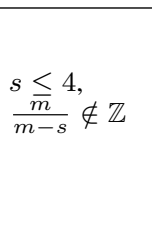 } & $s=1$ & infea. & $\infty$ \\
\hline & $s=2$ & infea. & $\infty$ \\
\hline & $s=3$, even $m$ & $\frac{m}{2}$ & $\frac{m}{2}$ \\
\hline & $s=3$, odd $m$ & infea. & $\infty$ \\
\hline & $s=4$, even $m$ & $\frac{3 m}{8}$ & $3\left\lfloor\frac{m}{8}\right\rfloor+\frac{m_{8}}{2}$ \\
\hline & $s=4$, odd $m$ & infea. & $\infty$ \\
\hline \multirow{3}{*}{$\begin{array}{l}s \geq m-3, \\
\frac{m}{m-s} \notin \mathbb{Z}\end{array}$} & $m-s=2$, odd $m$ & \multirow{3}{*}{$\begin{array}{l}\text { infea. } \\
\frac{3}{2}+\frac{9}{2 s} \\
\frac{3}{2}+\frac{9}{2 s}\end{array}$} & $\infty$ \\
\hline & $m-s=3$, even $m$ & & 3 \\
\hline & $m-s=3$, odd $m$ & & 4 \\
\hline
\end{tabular}

\section{CONCLUSION}

In this paper we studied the secure decentralized PICOD with circular side information at the users. We first proved that some settings, unknown in the literature priori to this work, are infeasible. For all the remaining cases, we proposes achievable schemes that use the same number of transmissions as predicted by our converse bound under the constraint of linear encoding up to a constant additive gap. Ongoing work includes extending the setting to other type of side information structures.

\section{REFERENCES}

[1] M. W. Alexandra Porter, "Embedded index coding," Information Theory Workshop, 2019. arXiv:1904.02179.

[2] Z. Bar-Yossef, Y. Birk, T. S. Jayram, and T. Kol, "Index coding with side information," IEEE Trans. on Information Theory, vol. 57, no. 3, pp. 1479-1494, Mar 2011.

[3] S. Brahma and C. Fragouli, "Pliable index coding," IEEE Trans. on Information Theory, vol. 61, no. 11, pp. 6192-6203, Nov 2015.

[4] T. Liu and D. Tuninetti, "Decentralized pliable index coding," Proc. Int. Symp. Inf. Theory, 2019.

[5] - "Private pliable index coding," arXiv:1904.04468, 2019.

[6] - "Secure decentralized pliable index coding," Proc. Int. Symp. Inf. Theory, 2020.

[7] V. Narayanan, J. Ravi, V. K. Mishra, B. K. Dey, N. Karamchandani, and V. M. Prabhakaran, "Private index coding," Proc. Int. Symp. Inf. Theory, 2018.

[8] S. Sasi and B. S. Rajan, "On pliable index coding," arXiv:1901.05809, 2019.

[9] L. Song and C. Fragouli, "A deterministic algorithm for pliable index coding," in Proc. Int. Symp. Inf. Theory, July 2016. 


\section{APPENDIX A}

CASE $m>2 s, m-4 \geq s \geq 5$

We show that for all cases in this region, there exists a scheme that achieves the converse bound within a constant gap. In this region our converse bound is $3 m / 2 s$. In order to achieve the optimality, we need to have the achievable scheme satisfy roughly $2 s / 3$ users in each transmission. We therefore propose the following scheme.

\section{A. Achievable schemes}

1) Scheme 1 satisfies $2 s$ users: One round of scheme 1 consists of 3 transmissions:

1) $w_{i}+w_{i+1}$,

2) $w_{i+3}+w_{i+s}$

3) $w_{i+s+1}+w_{i+s+2}$.

Here $i$ is the index of the first user among the $2 s$ users that are satisfied by the 3 transmissions. The $2 s$ satisfied users are $u_{i}, U_{[i+2: i+2 s-1], u_{i+2 s+1}}$. The transmissions and the satisfied users are shown in Fig. 7 7 As we can see the satisfied users can be seen in three groups. The two groups on the left and right contain one user each, while the middle group contains $2 s-2$ users. The scheme can be used repeatedly. The number of users that are satisfied is thus $2 k s$ where $k \in \mathbb{Z}^{+}$.

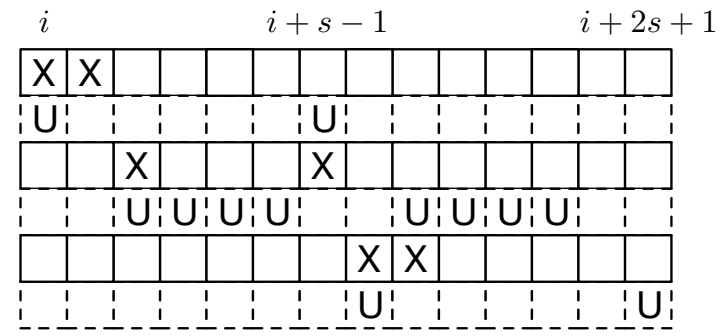

Fig. 7: Scheme 1.

Scheme 1 satisfies $2 s$ users using 3 transmissions. The messages that are involved in one transmission of scheme 1 do not appear in the other transmissions of scheme 1. Therefore, all transmissions in all rounds of scheme 1 satisfy the security constraint.

Note that on average each transmission satisfies $2 s / 3$ users in this scheme. This scheme is therefore optimal under the linear encoding constraint. If $\frac{m}{2 s} \in \mathbb{Z}$ this is the linear optimal scheme that achieves $\ell^{*}=\frac{3 m}{2 s}$.

The schemes in the rest of this section are proposed to address the cases where $\frac{m}{2 s} \notin \mathbb{Z}$ and $m>2 s$.

2) Scheme 2 for $2 s+2$ users: Scheme 2 satisfies $2 s+2$ users using 4 transmissions. Let $i$ be the index of the first users that are satisfied. The 4 transmissions are

1) $w_{i-\left(\left\lceil\frac{1-s}{2}\right\rceil-1\right)}+\cdots+w_{i+1}$,

2) $w_{i+2}+w_{i+\left\lceil\frac{s+1}{2}\right\rceil-1}$,

3) $w_{i+\left\lceil\frac{s+1}{2}\right\rceil}+w_{i+s+2}^{2}$,

4) $w_{i+s+3}+\cdots+w_{i+s+\left\lceil\frac{s+1}{2}\right\rceil}$.

The satisfied users are $u_{i}, U_{[i+2: i+2 s+1], u_{i+2 s+3}}$. The transmissions and the satisfied users are shown in Fig. 8

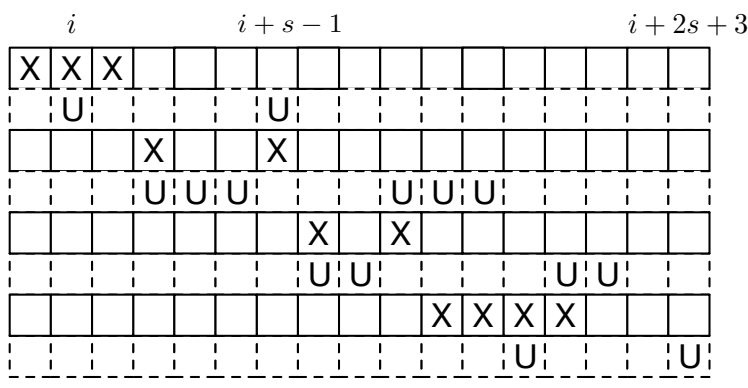

Fig. 8: Scheme 2.

3) Scheme 3 for $s+2$ users: Scheme 3 satisfies $s+2$ users using 3 transmissions. Let $i$ be the index of the first user satisfied by this scheme.

- When $s$ is even. The 3 transmissions are

1) $w_{i-s+3}+w_{i+2-s / 2}+w_{i+2-s / 2+1}+\cdots+w_{i}+w_{i+1}$,

2) $w_{i+1}+w_{i+2}+w_{i+3}$,

3) $w_{i+3}+w_{i+4}+\cdots+w_{i+s / 2+2}+w_{i+s+1}$.

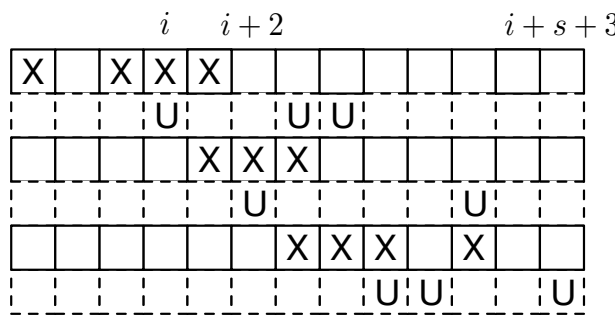

Fig. 9: Scheme 3 for even $s$.

- When $s$ is odd. The 3 transmissions are

1) $w_{i-s+4}+w_{i+2-(s-1) / 2}+w_{i+2-(s-1) / 2+1}+\cdots+$ $w_{i}+w_{i+1}$

2) $w_{i+1}+w_{i+2}+w_{i+4}$,

3) $w_{i+3}+w_{i+4}+\cdots+w_{i+(s+1) / 2+2}+w_{i+s+1}$.

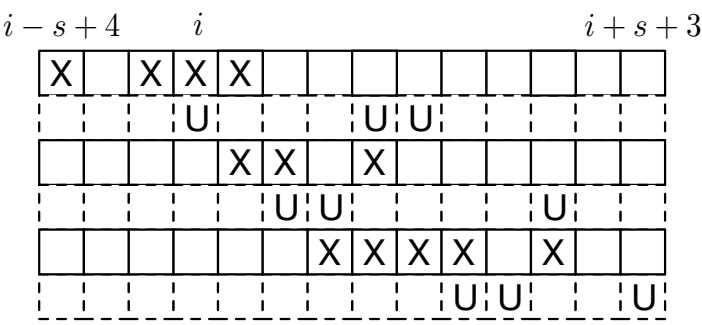

Fig. 10: Scheme 3 for odd $s$.

4) Scheme 4 for $n^{\prime}+2 \in\{2\} \cup[4: 2 s-2]$ users: Here $n^{\prime}$ is the number of satisfied users in the middle group.

- $n^{\prime}=0: 2$ users are satisfied by one transmission:

1) $w_{i-s+3}+\cdots+w_{i}+w_{i+1}$

- $n^{\prime} \in[2: s-2]: n^{\prime}+2$ users are satisfied by two transmissions:

1) $w_{i+\left\lceil n^{\prime} / 2\right\rceil-s}+w_{i+n^{\prime}-s+2}+w_{i+n^{\prime}-s+3}+\cdots+w_{i}+$ $w_{i+1}$. 


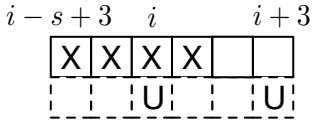

Fig. 11: Scheme $4, n^{\prime}=0$

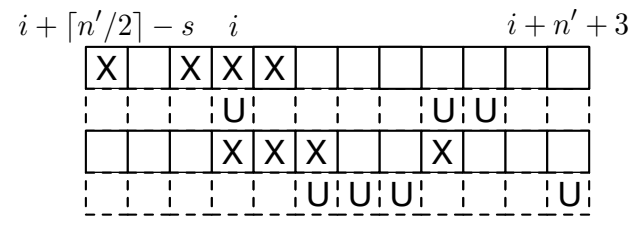

Fig. 12: Scheme 4, $n^{\prime} \in[2: s-2]$

2) $w_{i+n^{\prime}-s+3}+w_{i+n^{\prime}-s+4}+\cdots+w_{i+2}+w_{i+\left\lceil n^{\prime} / 2\right\rceil+2}$ - $n^{\prime}=s-1$ : s+1 users are satisfied by three transmissions:

1) $w_{i-s+3}+w_{i+\left\lfloor n^{\prime} / 2\right\rfloor-s+2}+w_{i+\left\lfloor n^{\prime} / 2\right\rfloor-s+3}+\cdots+$ $w_{i}+w_{i+1}$.

2) $w_{i}+w_{i+1}+w_{i+2}+w_{i+3}$.

3) $w_{i+2}+w_{i+3}+\cdots+w_{i+1+\left\lfloor n^{\prime} / 2\right\rfloor+1}+w_{i+1+n^{\prime}}$.

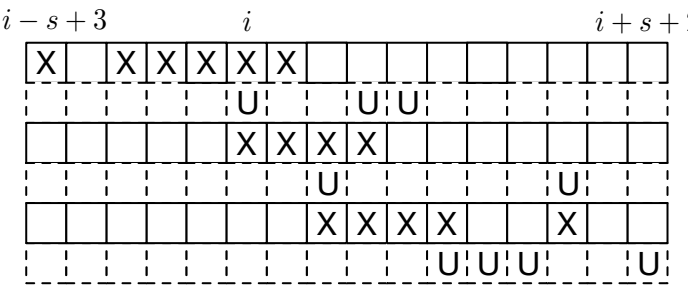

Fig. 13: Scheme $4, n^{\prime}=s-1$

- $n^{\prime}=s: \mathrm{s}+2$ users are satisfied by three transmissions:

1) $w_{i-s+3}+w_{i+\left\lfloor n^{\prime} / 2\right\rfloor-s+2}+w_{i+\left\lfloor n^{\prime} / 2\right\rfloor-s+3}+\cdots+$ $w_{i}+w_{i+1}$.

2) $w_{i+1}+w_{i+2}+w_{i+3}$.

3) $w_{i+3}+\cdots+w_{i+1+\left\lfloor n^{\prime} / 2\right\rfloor+1}+w_{i+1+n^{\prime}}$.

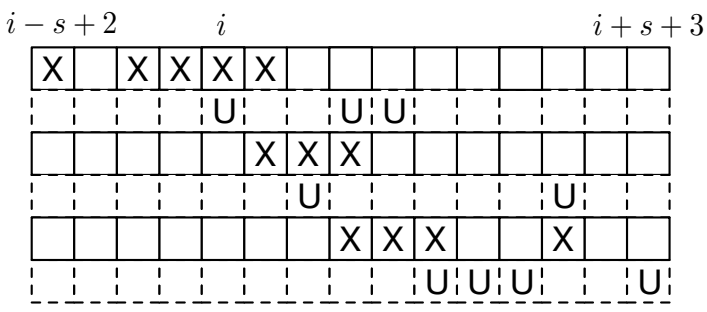

Fig. 14: Scheme $4, n^{\prime}=s$

- $n^{\prime} \in[s+1: 2 s-4]: n^{\prime}+2$ users are satisfied by three transmissions:

$$
\begin{aligned}
& \text { 1) } w_{i+1+n^{\prime}-s-s}+w_{i+\left\lfloor n^{\prime} / 2\right\rfloor-s+2}+w_{i+\left\lfloor n^{\prime} / 2\right\rfloor-s+3}+ \\
& \cdots+w_{i}+w_{i+1} \text {. } \\
& \text { 2) } w_{i+2}+w_{i+1+n^{\prime}-s} \text {. } \\
& \text { 3) } w_{i+1+n^{\prime}-s+1}+w_{i+1+n^{\prime}-s+2}+\cdots+ \\
& w_{i+1+\left\lfloor n^{\prime} / 2\right\rfloor+1}+w_{i+2+s} \text {. }
\end{aligned}
$$

\section{B. Decodability}

We the proposed schemes can satisfy all $m>2 s$ users in the system.

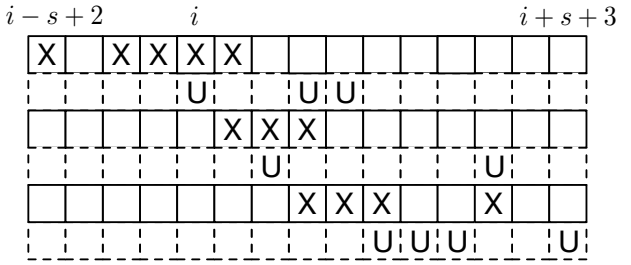

Fig. 15: Scheme 4, $n^{\prime} \in[s+1: 2 s-4]$

To achieve the converse bound $\ell \geq 3 m / 2 s$, we aim to satisfy $2 s / 3$ users in one transmission. Therefore, the intuition is to use scheme 1 as much as possible. Then we use schemes that involve a constant number of transmissions to satisfy the remaining users.

Note that scheme 1 satisfies $2 s$ users in each round, so the number of remaining users is less than $2 s$ after using scheme 1 . Scheme 4 can satisfy users of size 2 or $[4: 2 s-2]$. By applying scheme 4 after scheme 1 , we can deal with the cases where $m \bmod 2 s \in\{0,2,4,5, \ldots, 2 s-2\}$. For the case $m \bmod$ $2 s=2 s-1$, we use possibly scheme 1 , then scheme 2 and scheme 4 . For the cases $m$ mod $2 s \in\{1,3\}$, we use possibly scheme 1 , then scheme 3 and scheme 4.

The required numbers of transmissions are addressed as the follows.

- $m \bmod 2 s \neq 1,3,2 s-1$. In this case we use scheme $1 k$ times to satisfy $2 s k$ users. The remaining users are satisfied by scheme 4 . The total number of transmissions used is

$$
\ell= \begin{cases}3\left\lfloor\frac{m}{2 s}\right\rfloor+1 & m \bmod 2 s=2 \\ 3\left\lfloor\frac{m}{2 s}\right\rfloor+2 & m \bmod 2 s \in[4: s] \\ 3\left\lfloor\frac{m}{2 s}\right\rfloor+3 & m \bmod 2 s \in[s+1: 2 s-2]\end{cases}
$$

- $m \bmod 2 s=2 s-1$. In this case $m \geq 4 s-1$. We first use scheme 1 until the number of remaining users becomes $4 s-1$. We then use scheme 2 to satisfy $2 s+2$ users. The number of remaining users after applying scheme 2 is $2 s-3$. We then use scheme 4 to satisfy these $2 s-3$ users. The total number of transmissions is

$$
\ell=3 \frac{m+1}{2 s}+1=\frac{3 m}{2 s}+\frac{2 s+3}{2 s} .
$$

- $m \bmod 2 s=1$. In this case $m \geq 2 s+1$. We first use scheme 1 until the number of remaining users becomes $2 s+1$. We then use scheme 3 to satisfy $s+2$ users. The number of remaining users after applying scheme 3 is $s-1$. We then use scheme 4 to satisfy these $s-1$ users. The total number of transmissions is

$$
\ell=3 \frac{m-2 s-1}{2 s}+3+2=\frac{3 m}{2 s}+\frac{4 s-3}{2 s} .
$$

- $m \bmod 2 s=3$. In this case $m \geq 2 s+3$. We first use scheme 1 until the number of remaining users becomes $2 s+3$. We then use scheme 3 to satisfy $s+2$ users. The number of remaining users after applying scheme 2 is 
$s+1$. We then use scheme 4 to satisfy these $s+1$ users. The total number of transmissions is

$$
\ell=3 \frac{m-2 s-1}{2 s}+3+3=\frac{3 m}{2 s}+\frac{6 s-3}{2 s} .
$$

\section{Security constraint}

In this part we show that the proposed schemes are secure. By definition, the scheme is secure if all users can not decode any single message after decoding the desired messages. Since the encoding function is linear, the security constraint is equivalent to that there does not exist any standard basis in the row span of the submatrix of the generation matrix which is reduced by the columns which correspond to the interfering messages of a user.

We details the arguments for each scheme.

1) Scheme 1 satisfies $2 s$ users using 3 transmissions. The messages in different transmissions are all different. Therefore, the submatrix obtained from the columns of interfering messages of a user has all rows vectors orthogonal. None of the row vectors are standard basis. Therefore, the row span of the submatrix does not include any standard basis. The scheme 1 is thus secure. Note that it is also secure to repeat scheme 1 repeatedly since the row span of the submatrix obtained for one user does not contain any standard basis as well. This shows that the case $m \bmod 2 s=0$ can be satisfied securely using scheme 1 .

When followed by other schemes, the transmissions of scheme 1 also do not share any common messages to the transmissions of the other schemes. Therefore, the security holds for the case $m \bmod 2 s=2$ and $m \bmod$ $2 s \in[4: 2 s-2]$.

2) Scheme 2 satisfies $2 s+2$ users using 4 transmissions. Note that all 4 transmissions do not have common messages involved. By the same argument for scheme 1 , the $2 s+2$ users that are satisfied by scheme 2 will not decode more than one message by the transmissions in scheme 2.

When followed by scheme 4, there are exactly two transmissions which shared an exactly one message. Since the transmissions evolve strictly more than 2 messages, the interfering subspace does not contain any standard basis and the security constraint still holds.

3) Scheme 3 satisfies $s+2$ users using 3 transmissions. The 3 transmissions have at most one message in common pairwise. The 3 rows in the submatrix by the interfering messages contains at least 2 non-zero elements. Therefore, the linear combination of the rows can not be the standard basis. Therefore, the row span of the submatrix does not contain any standard basis. The $s+2$ users that are satisfied by scheme 3 will not decode more than one message by the transmissions in scheme 3 .

Again, when followed by scheme 4, one can check that the interfering subspace does not contain any standard basis. The user can not decode any message other than its desired message. The security constraint is satisfied.
4) Scheme 4 uses different number of transmissions for different subcases

- When $n^{\prime}=0,2$ users are satisfied by 1 transmission. It is thus secure.

- When $n^{\prime} \in[2: s-2]$, the scheme involves 2 transmissions. There are 2 messages in common of these 2 transmissions. Since each row contains at least 4 non-zero elements, any standard basis can not be a linear combination of the 2 rows.

- When $n^{\prime}=s-1$, the scheme involves 3 transmissions. There are at most 2 messages in common pairwise. Since each row contains at least 4 nonzero elements, any standard basis can not be a linear combination of the 3 rows.

- When $n^{\prime}=s$, the scheme involves 3 transmissions. There is at most 1 message in common pairwise. Since each row contains at least 3 non-zero elements, any standard basis can not be a linear combination of the 3 rows.

- When $n^{\prime} \in[s+1: 2 s-4]$, the scheme involves 3 transmissions. There is no message in common of these 3 transmissions. It is thus secure.

Overall, we conclude that no user is going to decode more than one message. The proposed scheme is secure.

\section{APPENDIX B}

$$
\text { CASE } s<m \leq 3 s / 2, m-4 \geq s \geq 5
$$

In this regime we consider another type of achievable scheme. We generate the codewords that satisfy the following two conditions:

1) After subtracting the messages in the side information set, each user observes one and only one transmission that contains only one message.

2) No common message between any two transmissions. That is, one message only appears in at most on transmission.

These two conditions guarantee the decodability as well as the security since linear combinations of the transmissions do not help to decode any new message. Therefore, the security per transmission provides the security of the whole transmissions.

\section{A. Case $m=k_{1}(2 p-2)+k_{2}(p)$}

For the simplicity of notation, define $p:=m-s$, which is the size of the complement of side information set. We first provide a basic structure of the codewords, which works for the case $m=k_{1}(2 m-2 s-2)+k_{2}(m-s)$, where $k_{1} \geq$ $2, k_{2} \geq 0, k_{1}, k_{2} \in \mathbb{Z}$.

The scheme takes 4 transmissions:

$$
\begin{aligned}
& \text { - } \sum_{i=1}^{p-1} w_{i}+\sum_{j=2}^{k_{1}+k_{2}-1} \sum_{i=1}^{p-1} w_{2 j(2 p-2)+i} . \\
& \text { - } w_{p}+w_{2 p-2}+\sum_{j=2}^{k_{1}-1}\left(w_{j(2 p-2)+p}+w_{(j+1)(2 p-2)}\right)+ \\
& \\
& \quad \sum_{j=0}^{k_{2}-1} w_{m-j p} . \\
& \text { - } \sum_{i=2 p-1}^{3 p-3} w_{i} . \\
& \text { - } w_{3 p-2}+w_{4 p-4} .
\end{aligned}
$$

The transmissions are illustrated in Fig. 16 . 


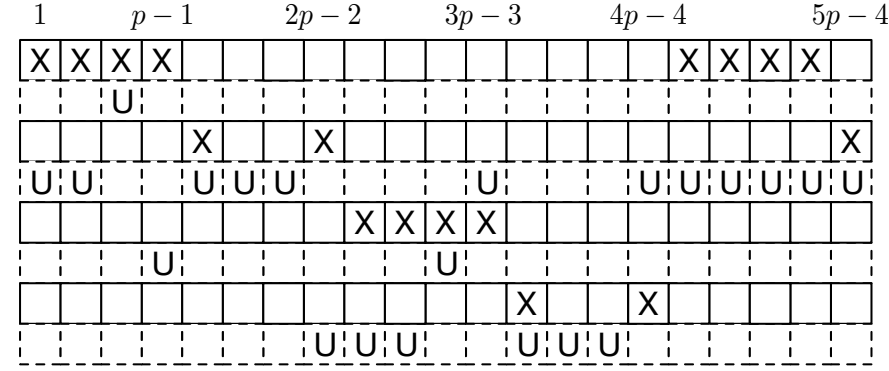

Fig. 16: Scheme for case $m=k_{1}(2 p-2)+k_{2}(p)$

By manipulating the parameters $k_{1}, k_{2}$, the scheme can satisfy all cases of $m \geq 4 p$ except the following cases:

- $m=5 p-1,5 p-2,7 p-3$.

- $m=5 p-3,6 p-3$.

\section{B. Case $m=k_{2}(2 p-2)+q$}

For the proposed scheme for case $m=k_{1}(2 p-2)+k_{2}(p)$ in Appendix B-A, call the part associated with $k_{1}$ part A, which consists of blocks of size $2 p-2$; the part associated with $k_{2}$ part $\mathrm{B}$, which consists of blocks of size $p$. We modify the scheme such that it works for $m=k_{2}(2 p-2)+q$, where $k_{1} \geq 1, p+2 \leq q \leq 2 p-2, k_{1}, q \in \mathbb{Z}$. The modification is done by shrink one block in part A to size $q$.

The scheme takes 4 transmissions:

- $w_{1}+w_{2 p-q}+\cdots+w_{p-1}+\sum_{j=1}^{k_{1}-1} \sum_{i=1}^{p-1} w_{j(2 p-2)+i}$.

- $w_{p}+w_{2 p-2}+\sum_{j=1}^{k_{1}-1}\left(w_{j(2 p-2)+p}+w_{j(2 p-2)+2 p-2}\right)$.

- $\sum_{i=m-q+1}^{m-p+1} w_{i}$.

- $w_{m-q+p}+w_{m}$.

The transmissions are illustrated in Fig. 17

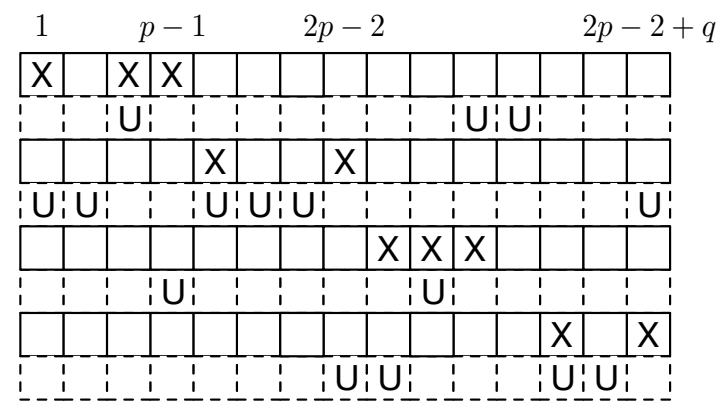

Fig. 17: Scheme for case $m=k_{2}(2 p-2)+q$

By taking $k_{1}=2, q=p+3, k_{1}=2, q=p+2, k_{1}=3, q=$ $p+3$, the cases $m=5 p-1,5 p-2,7 p-3$ can be solved using the this scheme. Moreover, by taking $k_{1}=1$, the case $3 p \leq m \leq 4 p-4$ can be solved by this scheme as well.

\section{Case by case study}

We are now left with the case $m=5 p-3,6 p-3$ and $m=4 p-3,4 p-2,4 p-1$. Since there are only 5 cases, we address them case by case.
1) $m=5 p-3$ : The scheme takes 3 transmissions:

- $\sum_{i=2}^{p-2} w_{i}+w_{p}+w_{2 p-2}+w_{4 p}$.

- $\sum_{i=2 p-1}^{2 p+1} w_{i}+w_{3 p+1}+w_{4 p-2}+w_{4 p-1}+w_{5 p-4}$.

- $w_{p-1}+w_{3 p-2}+w_{3 p}$.

The transmissions are illustrated in Fig. 18 .

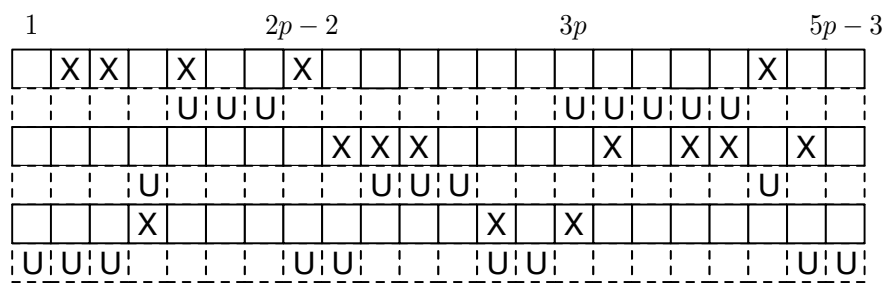

Fig. 18: Scheme for case $m=5 p-3$

2) $m=6 p-3:$ The scheme can be seen as modified scheme for the case $m=5 p-3$ by adding a block of size $p$. The scheme takes 3 transmissions:

- $\sum_{i=2}^{p-2} w_{i}+w_{p}+w_{2 p-2}+w_{3 p-2}+w_{5 p}$.

- $\sum_{j=0}^{1} \sum_{i=2 p-1}^{2 p+1} w_{i+j p}+w_{4 p+1}+w_{5 p-2}+w_{5 p-1}+w_{6 p-4}$.

- $w_{p-1}+w_{4 p-2}+w_{4 p}$.

The transmissions are illustrated in Fig. 19 .

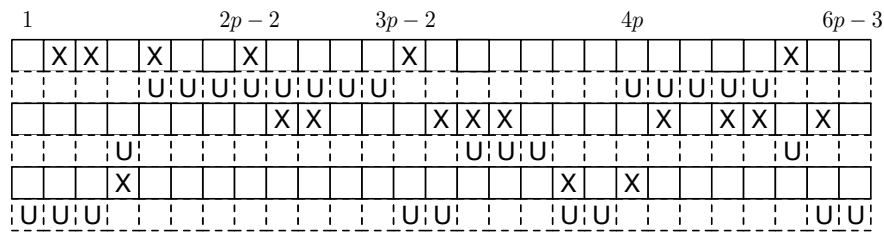

Fig. 19: Scheme for case $m=6 p-3$

3) $m=4 p-3$ : The scheme takes 3 transmissions:

- $w_{p-1}+w_{3 p-2}+w_{3 p-1}+w_{4 p-4}$ or $w_{p-1}+w_{3 p-2}+w_{3 p-1}$ if $p=4$.

- $\sum_{i=2}^{p-2} w_{i}+w_{p}+w_{2 p-2}+w_{3 p}$.

- $w_{2 p-1}+w_{2 p}$.

The transmissions are illustrated in Fig. 20.

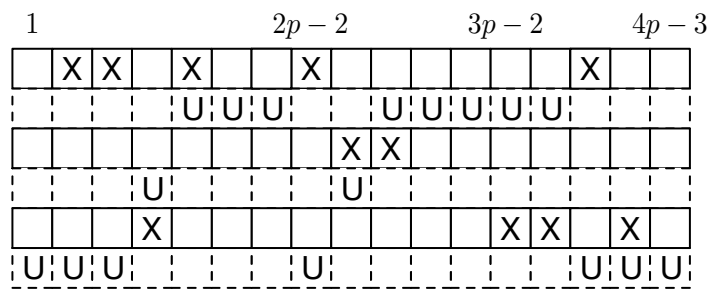

Fig. 20: Scheme for case $m=4 p-3$

4) $m=4 p-2$ : The scheme takes 3 transmissions:

- $w_{p-1}+w_{3 p-2}+w_{3 p}+w_{4 p-3}$ or $w_{p-1}+w_{3 p-2}+w_{3 p}$ if $p=4$.

- $\sum_{i=2}^{p-2} w_{i}+w_{p}+w_{2 p-2}+w_{3 p+1}$.

- $w_{2 p-1}+w_{2 p}+w_{2 p+1}$.

The transmissions are illustrated in Fig. 21 . 


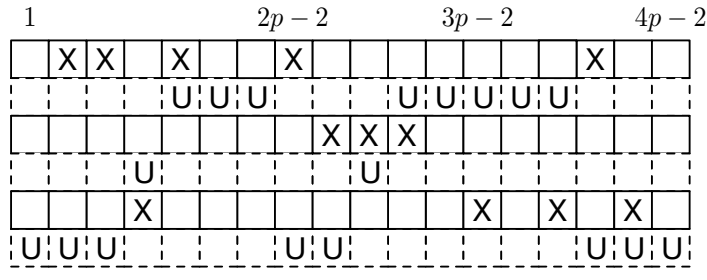

Fig. 21: Scheme for case $m=4 p-2$

5) $m=4 p-1$ : The scheme takes 3 transmissions:

- $w_{p-1}+w_{3 p-2}+w_{3 p+1}+w_{4 p-2}$ or $w_{p-1}+w_{3 p-2}+w_{3 p+1}$ if $p=4$.

- $\sum_{i=2}^{p-2} w_{i}+w_{p}+w_{2 p-2}+w_{3 p+2}$.

- $w_{2 p-1}+w_{2 p}+w_{2 p+1}+w_{2 p+2}$.

The transmissions are illustrated in Fig. 22

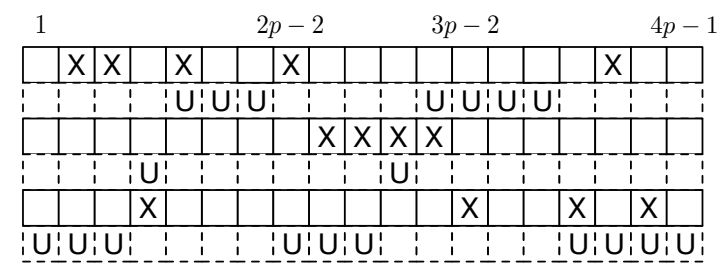

Fig. 22: Scheme for case $m=4 p-1$

Therefore, all the cases in the regime $s<m \leq 3 s / 2,4 \leq$ $s \leq m-5$ can be satisfied securely by the proposed scheme in this section using a finite number of transmissions. The linear converse bound can be achieved within an additive constant gap

\section{APPENDIX C \\ CASE $3 s / 2<m<2 s, m-4 \geq s \geq 5$}

In this regime we aim to satisfy $m$ users securely by a constant number of transmissions. The idea is to satisfy a small amount of the users at first such that the remaining users can be satisfied using the existing scheme securely. Specifically, we want to have the remaining users such that the schemes for the case $m \geq 2 s$ can be applied and the security constraint can be satisfied We use the notation $p:=m-s$ which is defined in Appendix B.

\section{A. Case $p \geq 7$}

The scheme we proposed can be split into two parts. The first part contains $a$ transmissions, where $a=2$ or 3 and will be decided later. The transmissions are $\sum_{i=1}^{s-1} w_{i}, \sum_{i=3}^{s+1} w_{i}$ when $a=2$ and $\sum_{i=1}^{s-1} w_{i}, \sum_{i=3}^{s+1} w_{i}, \sum_{i=5}^{s+3} w_{i}$ when $a=3$. Therefore, the first part satisfies $2 a$ users. The number of remaining users are $m-2 a$.

In the second part, we use the scheme that is similar to scheme 4 in Appendix A-A4 that has been used for case $m \geq$ $2 s$. The second part takes 3 transmissions as the follows.

- $w_{s+2 a}+w_{m-2}$.

- $w_{m-s-2}+\sum^{s+2 a-1}$

- $w_{m-s-2}+\sum_{i=a_{1}+\left\lceil\frac{m}{2}\right\rceil-1}^{s+2 a-1} w_{i}$.

- $\sum_{i=1}^{s+a-\left\lfloor\frac{m}{2}\right\rfloor-1} w_{i}+w_{2 s-m+2 a}+w_{m-1}+w_{m}$.
Note that the first transmission needs $m-2 \geq s+2 a+1$, which implies the condition $m-s=p \geq 2 a+3 \geq 7$. By the 3 transmissions, the second part satisfies all the remaining $m-2 a$ users. The schemes are illustrated in Fig 23 .

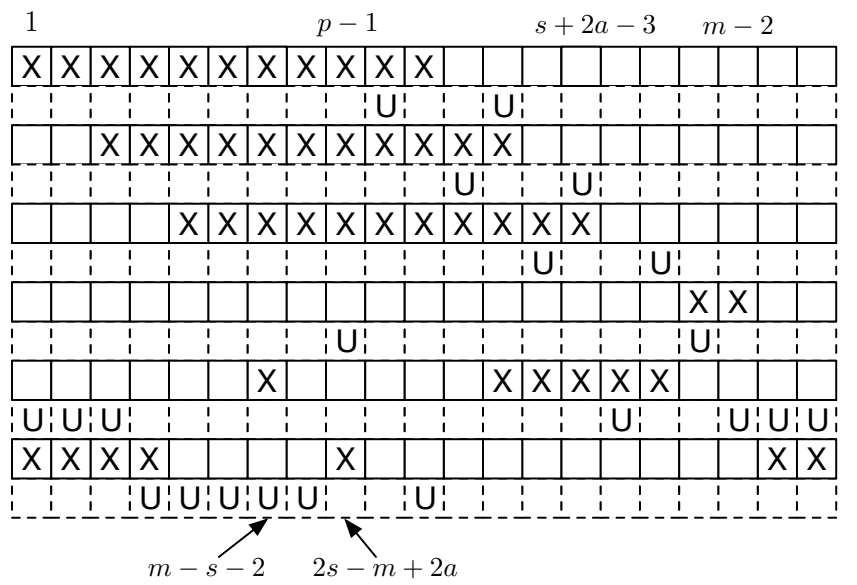

Fig. 23: Scheme for the case $3 s / 2<m<2 s$

We now decide the value of $a$, which will make the security constraint satisfied.

The security constraint can not be satisfied when the second part allows some users to decode more than one message. Note that the transmissions in the second part are the scheme used in Appendix A-A4 In Appendix A-A4 we have the condition $m>2 s$, thus the last two transmissions are guaranteed to not have common messages. However, when the scheme is used in the case $m<2 s$, it is possible that these two transmissions contain the common messages. The users can possibly decode an extra message after decoding their desired messages thus making the scheme possibly insecure.

Specifically, when $m-s-2=2 s-m+2 a$ the last two transmissions have $w_{m-s-2}$ as the common message. User $u_{s+a_{1}-\left\lfloor\frac{m}{2}\right\rfloor-1}$ has the desired message as $w_{m-s-2}$ and can decode $w_{m-s-2}$ by the last transmission. Note that $a_{1}+\left\lceil\frac{m}{2}\right\rceil-1-\left(s+a_{1}-\left\lfloor\frac{m}{2}\right\rfloor-1\right)=m-s=p$. That means that user $u_{s+a_{1}-\left\lfloor\frac{m}{2}\right\rfloor-1}$ can decode $w_{a_{1}+\left\lceil\frac{m}{2}\right\rceil-1}$ by the second transmission since there only two messages involved in the second transmission that are not in the side information set of user $u_{s+a_{1}-\left\lfloor\frac{m}{2}\right\rfloor-1}$ and one of the messages is its desired message. This violates the security constraint.

To secure the scheme, we choose $a$ such that $m-s-2 \neq$ $2 s-m+2 a$, that is, $a \neq p-s / 2-1$. By such choice of $a$ the scheme becomes secure. If we have the freedom to choose $a$ to be either 2 or 3 , the security constraint can be satisfied. Therefore, the scheme does not work only if $a$ can only be 2 and $p-s / 2-1=2$. That is, $7 \leq p<9$ and $p=s / 2+3$. Two cases are included:

1) $p=7, s=8, m=15$.

2) $p=8, s=10, m=18$.

a) Case $p=7, s=8, m=15$ : We propose a scheme with 6 transmissions that works for this case. The transmissions are: 
- $\sum_{i=1}^{7} w_{i}$

- $\sum_{i=3}^{9} w_{i}$.

- $\sum_{i=5}^{11} w_{i}$.

- $\sum_{i=7}^{13} w_{i}$.

- $w_{10}+w_{13}+w_{14}+w_{15}$

- $w_{1}+w_{3}+w_{14}+w_{15}$.

The transmissions are illustrated in Fig 24

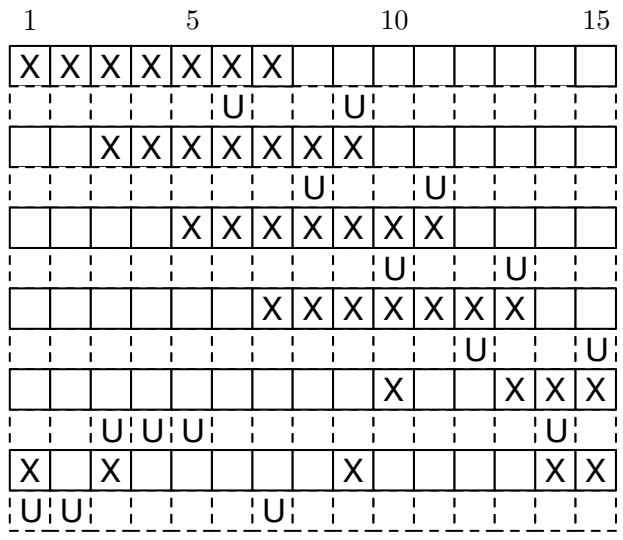

Fig. 24: Scheme for the case $p=7, s=8, m=15$

b) Case $p=8, s=10, m=18$ : Note that this is the case where $m$ and $s$ are even. By the result in [6] we know that there exists scheme with $m / 2$ transmissions that can securely satisfy all users. Since here we are only interested in a single case and we are satisfied by a scheme with a finite number of transmissions. The scheme with $m / 2$ transmissions, although suboptimal, is enough for us to claim that for the case $p=$ $8, s=10, m=18$ can be securely satisfied with a finite number of transmissions.

Therefore, for all cases with $p \geq 7$, we show the feasibility.

\section{B. Case $4 \leq p \leq 6$}

We now study the remaining cases in the regime $3 s / 2<$ $s<2 s, m-4 \geq s \geq 5$.

Note that in this regime we have $p<s<2 p$. Therefore, for $4 \leq p \leq 6$, the number of possible cases are finite. We address them cases by case.

a) Even $m$ : The cases with even $m$ include $p=4,6$, even $s$ and $p=5$, odd $m$. The largest $m$ in this case is $p+s=6+10=16$. By the result in [6], the scheme with $m / 2$ transmissions can securely satisfy these cases. The number of transmissions needed is upper bounded by $16 / 2=8$.

The remaining cases are the cases where $m$ is odd.

b) Case $p=4, s=5, m=9$ : We propose a scheme with 4 transmissions that works for this case. The transmissions are:

- $\sum_{i=1}^{4} w_{i}$.

- $\sum_{i=3}^{6} w_{i}$.

- $w_{5}+w_{7}+w_{8}$.

- $w_{1}+w_{8}+w_{9}$.

The transmissions are illustrated in Fig 25

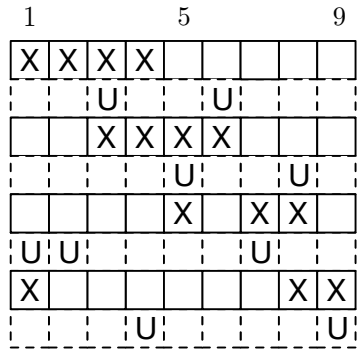

Fig. 25: Scheme for the case $p=4, s=5, m=9$

c) Case $p=4, s=7, m=11$ : We propose a scheme with 4 transmissions that works for this case. The transmissions are:

- $w_{2}+w_{4}+w_{6}$.

- $w_{5}+w_{6}+w_{7}+w_{9}$.

- $w_{1}+w_{7}+w_{8}+w_{9}+w_{10}+w_{11}$

- $w_{1}+w_{2}+w_{3}+w_{10}+w_{11}$.

The transmissions are illustrated in Fig 26.

\begin{tabular}{|c|c|c|c|c|c|c|c|c|c|}
\hline 1 & & & 5 & & & & & 10 & 11 \\
\hline & $X$ & & & $X$ & & & & & \\
\hline & & & U & & & & & : & \\
\hline & & & $X$ & $\mathrm{X}$ & $X$ & & $\bar{X}$ & & \\
\hline$U_{1}^{1}$ & & & & & $\mathrm{U}$ & $U:$ & & & \\
\hline $\mathrm{X}$ & & & & & $X$ & $\mathrm{X}$ & $X$ & $\mathrm{X}$ & $X$ \\
\hline & & & & & & & & & U \\
\hline $\mathrm{X}$ & $\mathrm{X}$ & & & & & & & $\mathrm{X}$ & $\mathrm{X}$ \\
\hline & $U_{1}^{\prime}$ & & & 1 & & & & & 1 \\
\hline
\end{tabular}

Fig. 26: Scheme for the case $p=4, s=7, m=11$

d) Case $p=5, s=6, m=11$ : We propose a scheme with 5 transmissions that works for this case. The transmissions are:

- $\sum_{i=1}^{5} w_{i}$.

- $\sum_{i=3}^{r} w_{i}$.

- $\sum_{i=5}^{9} w_{i}$

- $w_{7}+w_{9}+w_{10}+w_{11}$.

- $w_{1}+w_{2}+w_{11}$.

The transmissions are illustrated in Fig 27

\begin{tabular}{|c|c|c|c|c|c|c|c|c|c|c|}
\hline 1 & & & & 5 & & & & & & \\
\hline$X$ & $X$ & $X$ & $X$ & $X$ & & & & & & \\
\hline & & & $U$ & & & $U$ & & & & \\
\hline & & $\mathrm{X}$ & $\mathrm{X}$ & $X$ & $X$ & $X$ & & & & \\
\hline & & & & & $U$ & & & $U^{\prime}$ & & \\
\hline & & & & $\mathrm{X}$ & $X$ & $\mathrm{X}$ & $X$ & $\mathrm{X}$ & & \\
\hline & & & & & & & $U$ & & & $U_{1}^{\prime}$ \\
\hline & & & & & & $X$ & & $X$ & $x$ & $\mathrm{X}$ \\
\hline & $U$ & $U_{1}^{\prime}$ & & & & & & & U & \\
\hline $\mathrm{X}$ & $X$ & & & & & & & & & $X$ \\
\hline & & & & & & & & & & \\
\hline
\end{tabular}

Fig. 27: Scheme for the case $p=5, s=6, m=11$ 
e) Case $p=5, s=8, m=13$ : We propose a scheme with 5 transmissions that works for this case. The transmissions are:

- $\sum_{i=1}^{7} w_{i}$.

- $\sum_{i=3}^{9} w_{i}$.

- $\sum_{i=5}^{1=\overline{1}} w_{i}$.

- $w_{8}+w_{11}+w_{12}+w_{13}$.

- $w_{1}+w_{3}+w_{12}+w_{13}$.

The transmissions are illustrated in Fig 28

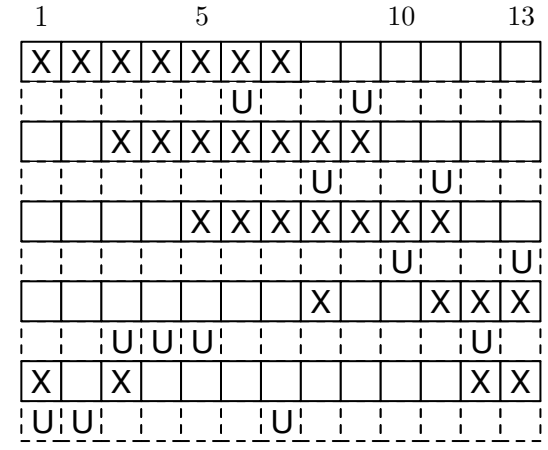

Fig. 28: Scheme for the case $p=5, s=8, m=13$

f) Case $p=6, s=7, m=13$ : We propose a scheme with 6 transmissions that works for this case. The transmissions are:

- $\sum_{i=1}^{6} w_{i}$.

- $\sum_{i=3}^{8} w_{i}$.

- $\sum_{i=5}^{10} w_{i}$.

- $\sum_{i=7}^{1 \overline{2}} w_{i}$.

- $w_{1}+w_{9}+w_{11}+w_{12}+w_{13}$

- $w_{1}+w_{2}+w_{3}+w_{12}+w_{13}$.

The transmissions are illustrated in Fig 29

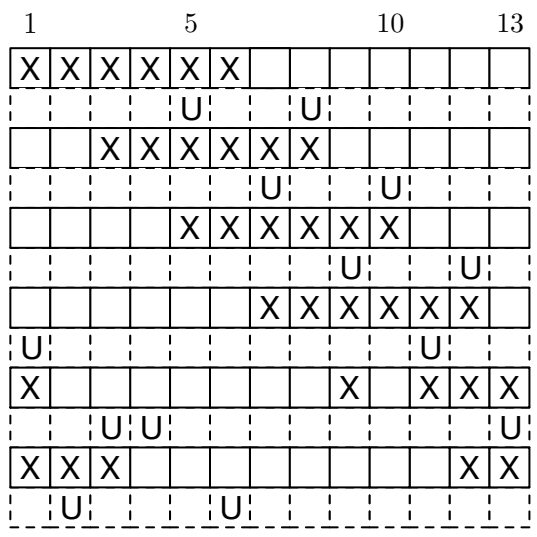

Fig. 29: Scheme for the case $p=6, s=7, m=13$

g) Case $p=6, s=9, m=15$ : We propose a scheme with 6 transmissions that works for this case. The transmissions are:

- $\sum_{i=1}^{8} w_{i}$.

- $\sum_{i=3}^{10} w_{i}$.

- $\sum_{i=5}^{12} w_{i}$.
- $\sum_{i=7}^{14} w_{i}$.

- $w_{1}+w_{10}+w_{13}+w_{14}+w_{15}$

- $w_{1}+w_{2}+w_{4}+w_{14}+w_{15}$.

The transmissions are illustrated in Fig 30

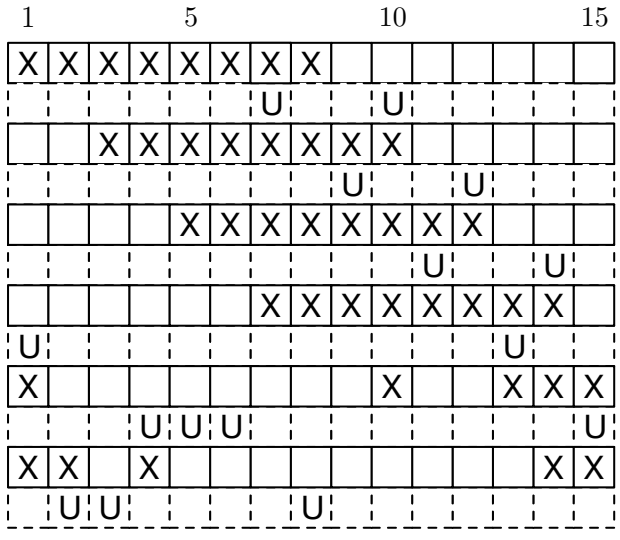

Fig. 30: Scheme for the case $p=6, s=9, m=15$

h) Case $p=6, s=11, m=17$ : We propose a scheme with 6 transmissions that works for this case. The transmissions are:

- $\sum_{i=1}^{10} w_{i}$.

- $\sum_{i=3}^{12} w_{i}$

- $\sum_{i=5}^{1 \overline{4}} w_{i}$

- $\sum_{i=7}^{1=5} w_{i}$

- $w_{1}+w_{1}+w_{15}+w_{16}+w_{17}$.

- $w_{1}+w_{2}+w_{5}+w_{16}+w_{17}$.

The transmissions are illustrated in Fig 31

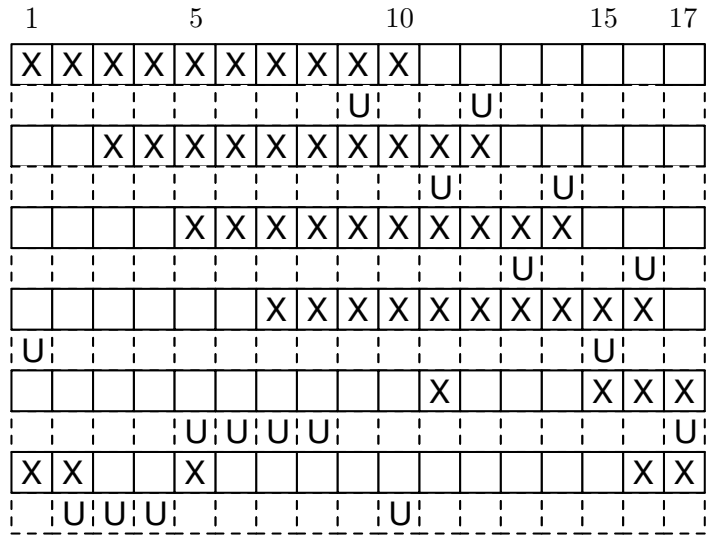

Fig. 31: Scheme for the case $p=6, s=11, m=17$

We address all cases in the regime where $3 s / 2<m<2 s$, $m-4 \geq s \geq 5$, and $4 \leq m-s \leq 6$ and show all cases are feasible. In this regime, the maximum number of transmissions needed is 9 for the case $m=18, s=10$.

\section{APPENDIX D}

CASE $s \leq 4$ OR $m-s=p \leq 3$

In this section we consider the cases where the size of the side information set $s$ is "small" or "large" when compared 
to the number of users $m$. These cases are studied separately because this regime contains all infeasible cases. Yet not all cases in this regime are infeasible. Specifically, the feasible cases include $s=3,4$, even $m$ and $s=m-3$.

A. $s=3$ and even $m$

In this case we use the scheme that takes $m / 2$ transmissions in [6]. This scheme is actually optimal in this case since $3 m / 2 s=m / 2$ in this case.

B. $s=4$ and even $m$

Note that $m \bmod 2 s \in\{0,2,4,6\}$ in this case. Therefore, by applying scheme 1 in Appendix A-A1 there will be 0 or 2 or 4 or 6 users remain. Then by scheme 4 in Appendix A-A4 we can satisfy the remain users with $0,1,2,3$ transmissions, respectively. Note that in this case the condition $s \geq 5$ on scheme 4 can be relaxed since the number of remaining users is always even so that the number of users need to be satisfied by scheme 4 will never be $s+1$. Also, note that in this case $s+1>2 s-4$, therefore the last subcase in scheme 4 will never happen.

C. $s=m-3$ and even $m$

3 transmissions can securely satisfy all users: $\sum_{i=1}^{s-1} w_{i}, \sum_{i=3}^{s+1} w_{i}, \sum_{i=5}^{m} w_{i}$. Note that by this scheme every user decodes one message, which is its desired message, and a sum of the other two messages that are not in its side information.

D. $s=m-3$ and odd $m$

Note that $m=5,7$ are infeasible as shown in Section IV. $m=9$ is the case $\frac{m}{m-s}=\frac{9}{3}=3 \in \mathbb{Z}$. Therefore, this case contains odd $m \geq 11$. We use the scheme for odd $m=s+$ 3 and $m \geq 11$ proposed in [6]. We satisfy all users by 4 transmissions. The 4 transmissions are $\left\{w_{1}+\sum_{i=1}^{\frac{m-7}{2}} w_{2 i}+\right.$ $w_{m-6}, \sum_{i=1}^{\frac{m-9}{2}} w_{2 i+1}+w_{m-5}+w_{m_{4}}, w_{m-3}+w_{m-2}, w_{m-1}+$ $\left.w_{m}\right\}$. 